ORIGINAL ARTICLE

\title{
Sensory level electrical muscle stimulation: effect on markers of muscle injury
}

\author{
T J McLoughlin, A R Snyder, P G Brolinson, F X Pizza
}

Br J Sports Med 2004;38:725-729. doi: 10.1136/bjsm.2003.007401

See end of article for authors' affiliations

.....................

Correspondence to: F X Pizza, University of Toledo, Toledo, Ohio 43606-3390, USA: fpizza@utnet.utoledo.edu

Accepted 20 October 2003
Background: Monophasic high voltage stimulation (MHVS) is widely prescribed for the treatment of inflammation associated with muscle injury. However, limited scientific evidence exists to support its purported benefits in humans.

Objective: To examine the efficacy of early initiation of MHVS treatment after muscle injury.

Methods: In a randomised, cross over design, 14 men performed repetitive eccentric contractions of the elbow flexor muscles followed by either MHVS or control treatment. MHVS treatments were applied five minutes and 3, 6, 24, 48, 72, 96, and 120 hours after eccentric contractions.

Results: MHVS resulted in a significant reduction $(p<0.05)$ in delayed onset muscle soreness 24 hours after eccentric exercise compared with controls. Elbow extension was significantly increased immediately after administration of MHVS compared with controls. No significant differences were observed between MHVS treatment and controls for maximal isometric strength, flexed arm angle, or arm volume.

Conclusions: Early and frequent application of MHVS may provide transient relief from delayed onset muscle soreness and short term improvements in range of motion after injurious exercise. However, MHVS treatment may not enhance recovery after muscle injury because of lack of improvements in strength and active range of motion.
$\mathrm{P}$ articipation in regular physical activity, especially involving unaccustomed eccentric contractions, may result in skeletal muscle injury. ${ }^{1}$ The effects of skeletal muscle injury, including decreased joint range of motion, decrements in force production, and soreness, may decrease sport related performance and exercise adherence, increase the risk of more severe injury, and impair performance of normal daily activities. The high incidence of skeletal muscle injury in the physically active population ${ }^{1}$ warrants investigation of effective intervention strategies to facilitate recuperation and attenuate negative side effects associated with physical activity.

Monophasic high voltage stimulation (MHVS) is widely prescribed for the treatment of muscle injury associated with injurious exercise. However, limited scientific evidence exists to support its use in treating skeletal muscle injury and associated acute inflammation in humans. Butterfield et $a l^{2}$ reported that MHVS applied 24, 48, and 72 hours after eccentric contractions did not influence delayed onset muscle soreness (DOMS) or loss of muscle function. The cascade of events associated with the acute inflammatory response has been shown to occur soon after the induction of injury. ${ }^{3}$ Specifically, blood neutrophil concentrations are increased within three hours and return to pre-exercise values by 24 hours after eccentric contractions. ${ }^{45}$ Therefore MHVS is probably best applied during the early phases of inflammation after acute muscle injury.

The purpose of this investigation was to examine the efficacy of early and frequent application of MHVS treatment after muscle injury. Maximal isometric strength, joint range of motion, DOMS, and limb volume were assessed and used as indicators of muscle injury. We hypothesised that early and frequent application of MHVS would result in more rapid restoration of markers of muscle injury.

\section{METHODS}

\section{Subjects}

Fourteen men (mean (SD) age 23.9 (4.6) years) with no history of musculoskeletal diseases, pre-existing inflammatory conditions, recent orthopaedic injuries, or participation in resistance arm exercise in the nine months preceding the study served as subjects. All provided written informed consent before participating in the study. Procedures were performed in accordance with guidelines established by the University of Toledo Human Subjects Committee.

\section{Experimental design}

In a randomised, cross over design, each subject participated in an experimental (EXP) and control (CT) condition, separated by a two week rest period. In the EXP condition, the elbow flexors were injured by repetitive eccentric contractions followed by treatment with sensory level MHVS (Rich-Mar, Inola, OK, USA) five minutes and 3, 6, $24,48,72,96$, and 120 hours after injury. In the CT condition, the contralateral limb underwent the same exercise protocol involving eccentric contractions, but the electrodes were placed on the injured limb at the treatment times described previously with no electrical current applied. For the CT condition, subjects were instructed that the treatment was a subsensory form of electrical treatment. Markers of muscle injury were assessed before and after treatment for all time periods across both conditions.

\section{Assessment of markers of muscle injury}

Markers of muscle injury were assessed in the following order for all time points. Flexed and relaxed arm angles were assessed by standard goniometry. ${ }^{6}$ Briefly, flexed and relaxed arm angles were assessed by instructing the subjects to maximally flex the elbow joint and to relax the arm at the side with the palm supinated respectively. DOMS was assessed during passive elbow flexion and extension using a visual analogue scale $(0-100 \mathrm{~mm})$. Arm volume was determined by a water displacement method modified from Griffin $e t \mathrm{al}^{7}$ and used as a gross indicator of muscle oedema.

Abbreviations: DOMS, delayed onset muscle soreness; MHVS, monophasic high voltage stimulation; TENS, transcutaneous electrical stimulation 

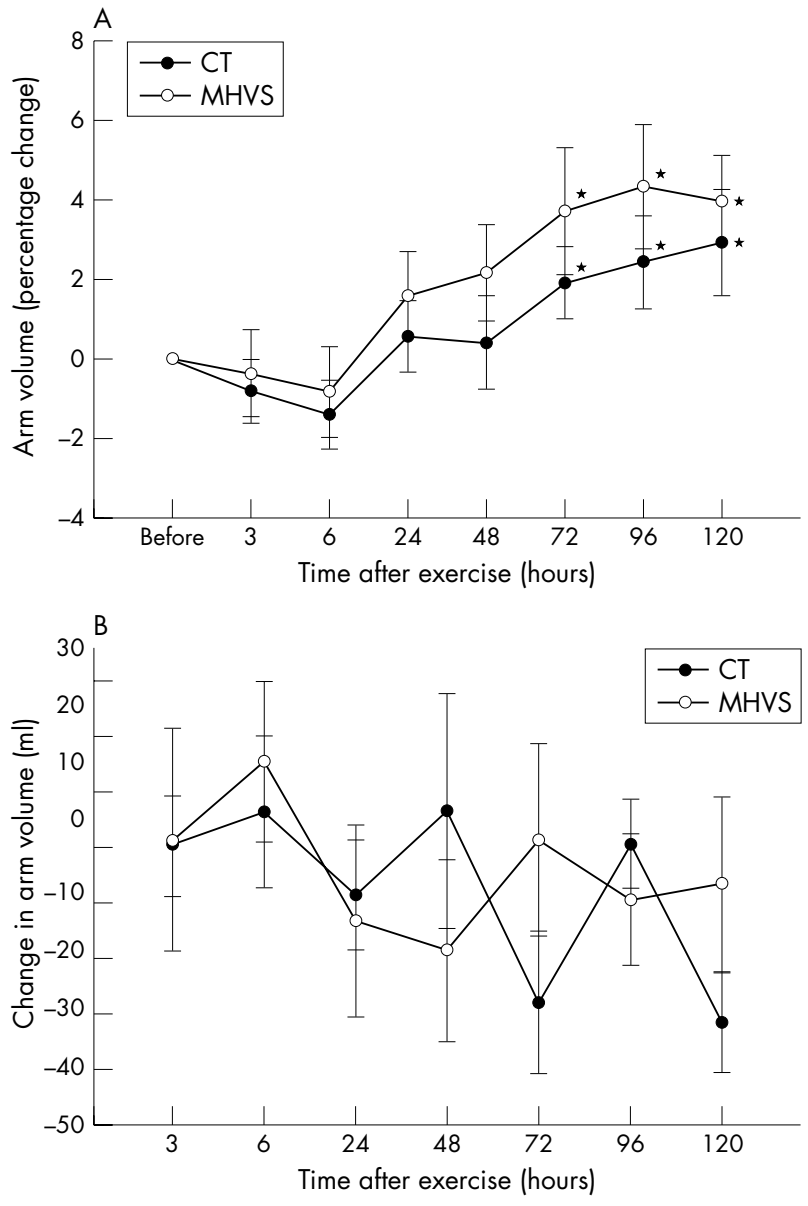

Figure 1 Effect of monophasic high voltage stimulation (MHVS) on arm volume after eccentric contractions. (A) Long term effect. Measurements were taken before the control (CT) or MHVS treatments at the respective time points (expressed as percentage change relative to measurement before the exercise (baseline measurement)). *Significantly different from the baseline measurement $(p<0.05)$. (B) Short term effect.

Measurements were taken $\sim 10$ minutes after the CT or MHVS

treatments and subtracted from the measurement before the treatment at the respective time points. All values are mean (SEM) $(n=14)$.

Finally, maximal isometric strength of the elbow flexor muscles was determined at $90^{\circ}$ of elbow flexion from a load cell interfaced with a microcomputer. All markers of muscle injury were assessed at all time points, except arm volume and DOMS, which were not assessed five minutes after the injury. About 20 minutes was required to complete the assessment of the selected markers of muscle injury.

Potential long and short term benefits of sensory level MHVS on the selected markers of muscle injury were assessed in the study. The long term effects-that is, day to day changes - of MHVS were determined by comparing measurements obtained before the stimulation treatments across all time points. Short term effects-that is, immediate changes observed after the 30 minute MHVS treatment-of MHVS were determined by subtracting measurements obtained before MHVS treatment from measurements obtained immediately after MHVS for each time point.

\section{Exercise protocol}

Each subject performed repetitive eccentric contractions for both the EXP and CT conditions as previously described. ${ }^{4}$ Briefly, subjects were seated in a modified arm curl bench with elbow positioned in full flexion while holding the
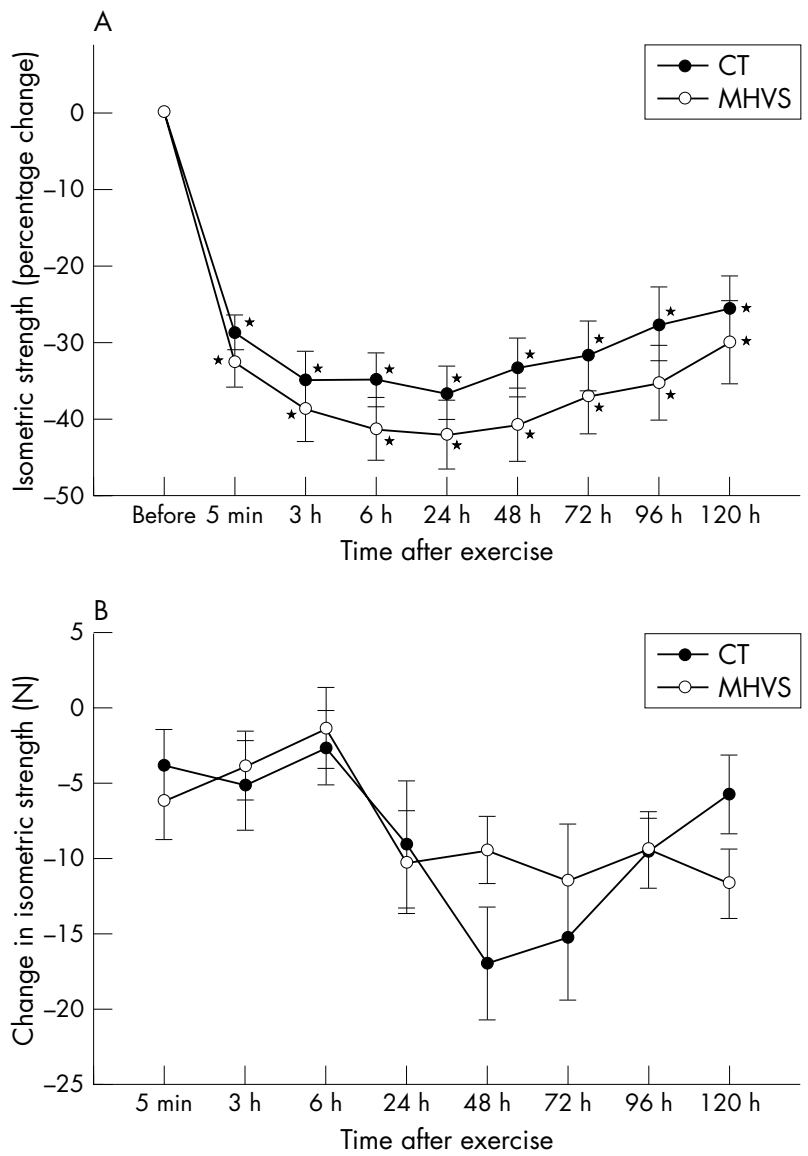

Figure 2 Effect of monophasic high voltage stimulation (MHVS) on maximal isometric strength after eccentric contractions. (A) Long term effect. Measurements were taken before the control (CT) or MHVS treatments at the respective time points (expressed as percentage change relative to measurement before the exercise (baseline measurement)). *Significantly different from the baseline measurement $(p<0.05)$.

(B) Short term effect. Measurements were taken 15 minutes after the CT or MHVS treatments and subtracted from the measurement before the treatment at the respective time points. All values are mean (SEM) ( $n=14)$.

handgrip. Subjects were instructed to maximally resist as the investigator forcibly extended the elbow joint for a period of four seconds. When full extension was reached, the investigator repositioned the subject's elbow in the flexed position. This sequence was repeated until a total of 25 eccentric contractions were completed.

\section{Electrical stimulation protocol}

MHVS was applied at the sensory level to the EXP limb after measurements of muscle injury. In accordance with common clinical practice, treatments were 30 minutes long consisting of continuous mode stimulation (negative polarity) with pulse frequency and pulse duration of 120 pulses/s and $100 \mu$ s respectively. A total of four active electrodes (two channels) located on the anterior portion of the distal aspect of the forearm (positive electrode), the medial portion of the proximal aspect of the forearm (negative electrode), the anterior portion of the distal aspect of the biceps brachii (negative electrode), and over the bicipital groove were placed on the limb (positive electrode). No significant differences were observed in the amount of electrical current administered across the various time points for the EXP condition (data not shown). 

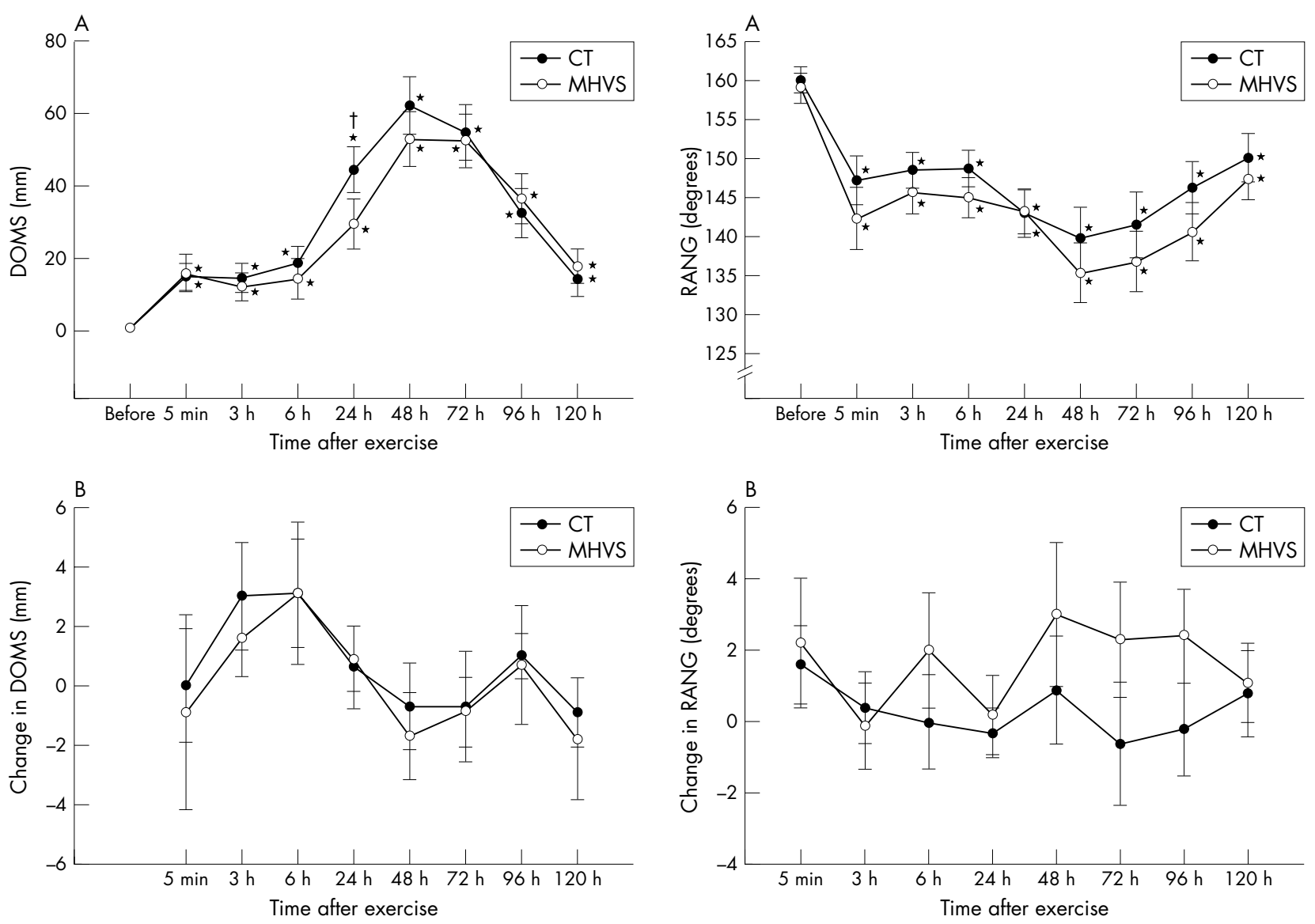

Figure 3 Effect of monophasic high voltage stimulation (MHVS) on delayed onset muscle soreness (DOMS) after eccentric contractions. (A) Long term effect. Measurements were taken before the control (CT) or MHVS treatments at the respective time points. *Significantly different from the baseline measurement $(p<0.05)$. †Significant difference between $\mathrm{CT}$ and MHVS at the respective time point (interaction; $\mathrm{p}<0.05)$. (B) Short term effect. Measurements were taken immediately after the CT or MHVS treatments and subtracted from the measurements before treatment at the respective time points. All values are mean (SEM) ( $n=14)$.

\section{Statistical analysis}

A two way repeated measures analysis of variance was used to determine main effects and interactions for the dependent variables: arm volume, maximal isometric strength, relaxed and flexed arm angles, and DOMS. A Student-NewmanKeuls post hoc test was used to locate differences between observed means for all variables. A probability level of 0.05 was used for determination of significant differences for all analyses. All data are represented as mean (SEM).

\section{RESULTS}

\section{Arm volume}

Arm volume was significantly increased at 72, 96, and $120 \mathrm{~h}$ after muscle injury in both EXP and CT conditions (fig 1A), indicating increased oedema in the days after injurious muscle contractions. However, MHVS had neither long nor short term effects (fig $1 \mathrm{~A}$ and $\mathrm{IB}$ respectively) on arm volume.

\section{Maximal isometric strength}

Maximal isometric strength was significantly decreased immediately after repetitive eccentric contractions relative to baseline values (before the eccentric contractions), and remained below baseline values throughout the treatment period (fig 2A). However, MHVS had neither long nor short

Figure 4 Effect of monophasic high voltage stimulation (MHVS) on relaxed arm angle (RANG) after eccentric contractions. (A) Long term effect. Measurements were taken before the control (CT) or MHVS treatments at the respective time points. *Significantly different from the baseline measurement $(p<0.05)$. (B) Short term effect. Measurements were taken about five minutes after the CT or MHVS treatments and subtracted from the measurements before treatment at the respective time points. There was a significant difference between control and MHVS across all time points $(p<0.05)$. All values are mean $(S E M)(n=14)$.

term effects (fig 2A and 2B respectively) on maximal isometric strength.

\section{Perceived soreness}

DOMS was significantly increased at all time points relative to the baseline values, regardless of treatment administered (fig 3A). Application of MHVS resulted in a significant reduction in DOMS relative to CT at 24 hours with a tendency to reduction at 48 hours $(p<0.072)$. No short term effect on DOMS was shown through MHVS treatment at any time point assessed (fig 3B).

\section{Range of motion}

Relaxed arm angle was significantly decreased and flexed arm angle was significantly increased at all time points relative to the baseline values, regardless of the treatment condition (fig $4 \mathrm{~A}$ and $5 \mathrm{~A}$ respectively). Thus range of motion was significantly reduced in the hours to days after repetitive eccentric contractions, which was not improved with MHVS treatment. MHVS had a significant acute effect on relaxed arm angle compared with CT (statistical main effect; fig 4B), but no differences were observed between MHVS and CT at any specific time point. In contrast, MHVS did not have a significant short term effect on flexed arm angle at any time point assessed (fig 5B). 

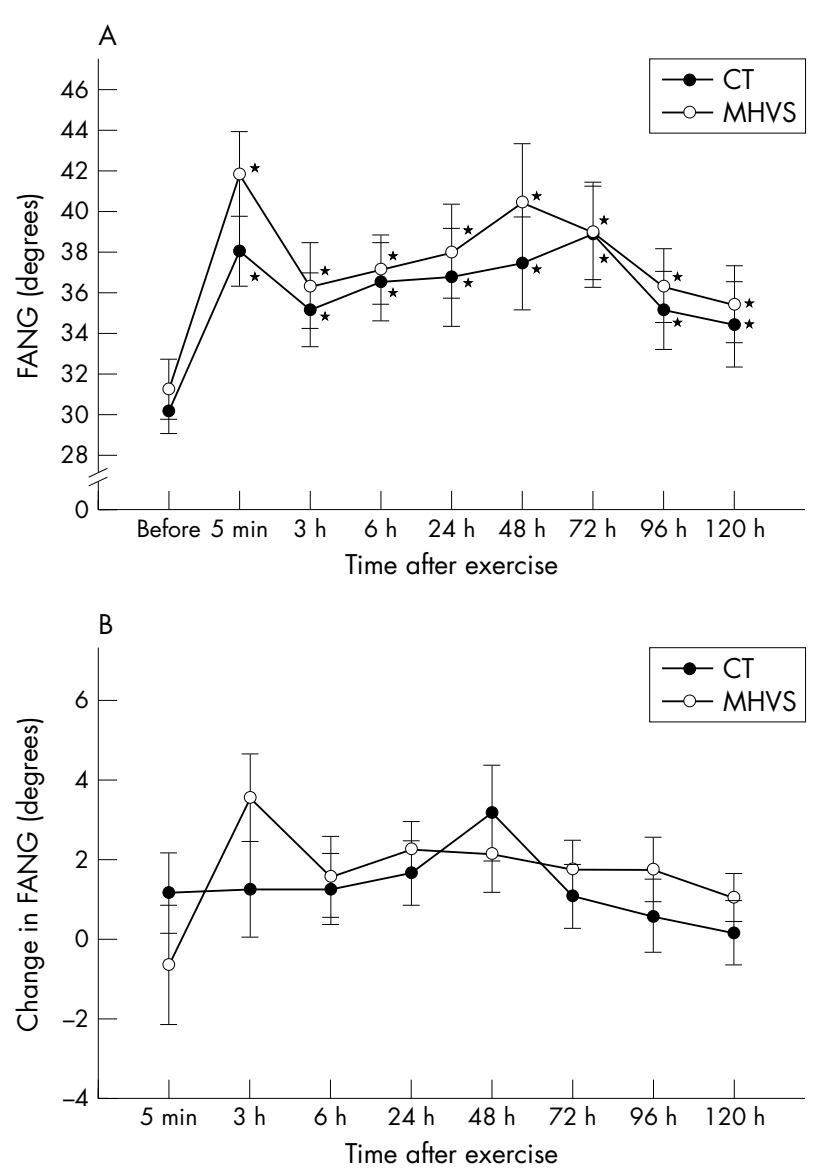

Figure 5 Effect of monophasic high voltage stimulation (MHVS) on flexed arm angle (FANG) after eccentric contractions. (A) Long term effect. Measurements were taken before the control (CT) or MHVS treatments at the respective time points. *Significantly different from the baseline measurement $(p<0.05)$. (B) Short term effect. Measurements were taken about five minutes after the CT or MHVS treatments and subtracted from the measurements before treatment at the respective time points. All values are mean (SEM) $(n=14)$.

\section{DISCUSSION}

The key findings in this study were the reductions in DOMS and the improvement in relaxed arm angle with MHVS treatment after eccentric contractions. These improvements were short lived, however. Specifically, MHVS relieved soreness 24 hours after injury, with a tendency to decreased soreness 48 hours after injury, compared with CT (fig 3A). The increased relaxed arm angle occurred only soon after MHVS treatment (fig 4B) as there were no long term improvements in relaxed arm angle after MHVS treatment. MHVS had no apparent short or long term influence on arm volume (fig 1), isometric strength (fig 2), or active range of motion (fig 5) after eccentric contractions. These findings indicate that aggressive application of MHVS may provide transient therapeutic benefits after muscle injury. However, considering that MHVS does not appear to result in functional improvements-for example, isometric strength and active range of motion-caution must be exercised about returning injured patients to physical activity after MHVS treatment.

Because muscle soreness can negatively affect the ability to perform activities of daily life, clinicians often focus on pain management when treating muscle injuries. Although electrotherapy is widely prescribed for the treatment of pain associated with injured muscle, studies on the potential effects of electrical stimulation on the transmission of noxious impulses are limited. Most research in this area has focused on the use of transcutaneous electrical stimulation (TENS), a specific electrotherapeutic approach to pain control entailing the transmission of a mild electrical current through electrodes strategically positioned around the site of injury. ${ }^{8}$ Although TENS units typically use a biphasic pulsed current compared with the monophasic current associated with MHVS, the electrical variables associated with sensory level TENS-for example, electrical intensity, pulse frequency, and pulse duration-resemble the sensory level MHVS protocol used in this study.

TENS has been shown to be effective in attenuating pain perception after injury in humans. ${ }^{910}$ Although the precise mechanism(s) responsible for mediating the therapeutic effects of TENS is not known, it is generally believed that it decreases pain perception by inhibiting afferent transmission of noxious impulses to higher brain centres. ${ }^{11}$ Garrison and Foreman $^{11}$ showed that TENS treatment, using variables similar to those used in this study (frequency, 100 pulses/s; pulse duration $100 \mu$ s), reduced noxious nerve transmission associated with mechanical compression of the hind limb in cats. Whether a similar mechanism(s) is responsible for mediating the reduction in pain perception elicited by MHVS treatment after muscle injury in this study remains to be elucidated. Interestingly, the reduction in DOMS with MHVS treatment occurred at the time when soreness was reaching its peak-that is, 24-48 hours after eccentric contractions; $\mathrm{p}<0.072$ for 48 hours compared with CT. Although the mechanism mediating this response cannot be elucidated from this study, it may be that a certain threshold level of soreness must be reached before the analgesic effects of sensory level stimulation can be manifested.

Loss of range of motion is a common consequence of muscle injury. In accordance, relaxed arm angle was decreased and flexed arm angle was increased (fig 4A and $5 \mathrm{~A}$ respectively) in the hours to days after eccentric contractions. Interestingly, 30 minutes of MHVS treatment resulted in a short term increase in relaxed arm angle after eccentric contractions (fig 4B). Similarly, Craig et al ${ }^{12}$ showed that high frequency TENS resulted in improvements in relaxed arm angle after muscle injury compared with placebo and control groups. In contrast, Denegar and Perrin ${ }^{10}$ showed that sensory level TENS treatment did not significantly influence relaxed arm angle after muscle injury. The discrepancy in findings across the various studies are not easily reconciled. However, differences in treatment protocols and severity of muscle injury may account for the varied findings.

Clarkson $e a^{13}$ suggested that a decrease in relaxed arm angle immediately after injurious exercise can be attributed to shortening of the connective tissue or muscle fibres themselves. The spontaneous muscle shortening, however, does not appear to be the result of active muscle contraction, as resting electromyographic activity is not increased after muscle injury. ${ }^{14}{ }^{15}$ Other possible explanations include the accumulation of $\mathrm{Ca}^{2+}$ within the muscle cell and/or mechanoreceptor involvement. ${ }^{13}{ }^{15}$ It is possible that MHVS activates voltage gated membrane channels resulting in a temporary restoration of calcium homoeostasis in the muscle cell and/or it excites nerve fibres associated with golgi tendon organs, thus activating a protective lengthening contraction. In theory, both of these mechanisms would elicit a transient increase in relaxed arm angle after muscle injury.

Despite the improvement in relaxed arm angle and reductions in DOMS after eccentric contractions, MHVS treatment had no short or long term influence on arm volume (fig 1), isometric strength (fig 2), or flexed arm angle (fig 5). For instance, Butterfield et $a l^{2}$ showed that MHVS applied in the days after eccentric contractions did not improve active 


\section{What is already known on this topic}

MHVS has been reported to be effective in reducing oedema, attenuating inflammatory cell invasion, and improving range of motion after muscle injury in various animal studies. However, limited scientific evidence exists to support the purported benefits in humans.

\section{What this study adds}

This study shows that early and frequent application of MHVS provides transient improvements in range of motion and attenuates muscle soreness after eccentric contractions in humans. Further studies are needed to ascertain whether these improvements translate into functionally significant outcomes in rehabilitation.

range of motion or muscle function in humans. The implication is that perceived pain and loss of passive range of motion associated with contraction induced muscle injury may be modestly improved with MHVS, but recovery of strength and active range of motion is not improved. Considering that the restoration of full active range of motion and strength are critical for complete rehabilitation of muscle and are probably greater determinants of recovery than are modest improvements in pain and passive range of motion, the lack of improvement in these outcomes with MHVS may not permit faster recovery after muscle injury due to an acute bout of eccentric exercise.

Despite the ubiquitous use of MHVS for the treatment of the sequelae associated with muscle injury, evidence supporting its efficacy is lacking. To our knowledge, this study shows for the first time that early and frequent application of sensory level MHVS attenuates some signs and symptoms associated with contraction induced muscle injury in humans. Specifically, chronic perception of pain was decreased in the days after muscle injury and passive range of motion was transiently increased soon after MHVS treatments. Thus MHVS may be a viable treatment for providing analgesic benefits and short term improvements in range of motion after injurious exercise. However, considering the transient nature and relatively small magnitude of improvement in relaxed arm angle and DOMS observed, further studies need to be performed to ascertain whether these improvements translate into functionally significant outcomes in rehabilitation.

\section{ACKNOWLEDGEMENTS}

This project was supported through a doctoral student grant provided by the NATA Research \& Education Foundation (to TJM).

\section{Authors' affiliations}

T J McLoughlin, A R Snyder, F X Pizza, University of Toledo, Toledo, Ohio 43606-3390, USA

P G Brolinson, Northwest Ohio Center for Sports Medicine, Ohio, USA

Conflict of interest: none declared

\section{REFERENCES}

1 Hootman JM, Macera CA, Ainsworth BE, et al. Epidemiology of musculoskeletal injuries among sedentary and physically active adults. Med Sci Sports Exerc 2002;34:838-44.

2 Butterfield DL, Draper DO, Ricard MD, et al. The effects of high-volt pulsed current electrical stimulation on delayed onset muscle soreness. J Athl Train 1997;32:15-20.

3 Reed BV. Effect of high voltage pulsed electrical stimulation on microvascular permeability to plasma proteins. A possible mechanism in minimizing edema. Phys Ther 1988;68:491-5

4 Pizza FX, Baylies H, Mitchell JB. Adaptation to eccentric exercise: neutrophils and E-selectin during early recovery. Can J Appl Physiol 2001;26:245-53.

5 Cannon JG, Orencole SF, Fielding RA, et al. Acute phase response in exercise: interaction of age and vitamin $E$ on neutrophils and muscle enzyme release. Am J Physiol 1990;259:R1214-19.

6 Daniels L, Worthingham C. Muscle testing: techniques of manual examination. Philadelphia: WB Saunders, 1986

7 Griffin JW, Newsome LS, Stralka SW, et al. Reduction of chronic posttraumatic hand edema: a comparison of high voltage pulsed current, intermittent pneumatic compression, and placebo treatments. Phys Ther 1990:70:279-86

8 Starkey C. Therapeutic modalities, 2nd ed. Philadelphia: F A Davis Company, 1999.

9 Denegar CR, Perrin DH, Rogol AD, et al. Influence of transcutaneous electrical nerve stimulation on pain, range of motion, and serum cortisol concentration in females experiencing delayed onset muscle soreness. J Orthop Sports Phys Ther 1989;11:100-3.

10 Denegar CR, Perrin DH. Effect of transcutaneous electrical nerve stimulation, cold, and a combination treatment on pain, decreased range of motion, and strength loss associated with delayed onset muscle soreness. J Athl Train 1992;27:200-6.

11 Garrison DW, Foreman RD. Decreased activity of spontaneous and noxiously evoked dorsal horn cells during transcutaneous electrical nerve stimulation (TENS). Pain 1994;58:309-15.

12 Craig JA, Barron J, Walsh DM, et al. Lack of effect of combined low intensity laser therapy/phototherapy (CLILT) on delayed onset muscle soreness in humans. Lasers Surg Med 1999;24:223-30.

13 Clarkson PM, Nosaka K, Braun B. Muscle function after exercise-induced muscle damage and rapid adaptation. Med Sci Sports Exerc 1992;24:512-20

14 Jones DA, Newham DJ, Clarkson PM. Skeletal muscle stiffness and pain following eccentric exercise of the elbow flexors. Pain 1987:30:233-42.

15 Howell JN, Chila AG, Ford G, et al. An electromyographic study of elbow motion during postexercise muscle soreness. J Appl Physiol 1985;58:1713-18. 